\title{
ABELIAN VARIETIES DEFINED OVER THEIR FIELDS OF MODULI, I $\dagger$
}

\section{J. S. MILNE}

Whenever we consider a triple $(A, \mathscr{C}, \theta)$ we will mean that $A$ is an abelian variety of dimension $d, \mathscr{C}$ is a polarization of $A, \theta: F \rightarrow \operatorname{End}^{\circ}(A)=$ End $(A) \otimes_{\mathbf{Z}} \mathbf{Q}$ is a ring homomorphism where $F$ is a field of degree $2 d$ over $\mathbf{Q}, \theta(F)^{\prime}=\theta(F)$ where $\alpha \mapsto \alpha^{\prime}$ is the involution of $\operatorname{End}^{\circ}(A)$ induced by $\mathscr{C}$, and that $A, \mathscr{C}$, and $\theta$ are all defined over some subfield of the complex numbers $C$. $F$ is then necessarily a $C M$-field, and $(A, \mathscr{C}, \theta)$ is of type $(F, \Phi ; \mathfrak{a}, \zeta)$ in the sense of $[5$, p. 128] for some lattice $\mathfrak{a}$ in $F$ and element $\zeta$ of $F$. We will assume that the reader is familiar with the definitions in [5].

Our main result is that $(A, \mathscr{C}, \theta)$ always has a model defined over its field of moduli $k_{0}$, i.e. that there is an $\left(A_{0}, \mathscr{C}_{0}, \theta_{0}\right)$ defined over $k_{0}$ which becomes isomorphic to $(A, \mathscr{C}, \theta)$ over $\mathbf{C}$. As a consequence, one gets an alternative proof of a theorem of Casselman's [6, Theorem 6] characterizing those Grössen-characters which arise from abelian varieties. Also, one obtains a positive answer to a question of Shimura's concerning the existence of such Grössen-characters [6, p. 513].

In a second paper we intend to consider the question of, given $(A, \mathscr{C}, \theta)$, when is the pair $(A, \mathscr{C})$ defined over its field of moduli.

We write $k_{a b}$ for the maximal abelian extension of a field $k$, and $k$ for its algebraic closure. $\left(F^{\prime}, \Phi^{\prime}\right)$ denotes the reflex of a $C M$-type $(F, \Phi)$.

THEOREM. Let $(A, \mathscr{C}, \theta)$, as above, be of $C M$-type $(F, \Phi)$. Then there is a model $\left(A_{0}, \mathscr{C}_{0}, \theta_{0}\right)$ of $(A, \mathscr{C}, \theta)$ defined over the field of moduli $k_{0}$ of $(A, \mathscr{C}, \theta)$ and such that all torsion points of $A_{0}$ are rational over $F^{\prime}{ }_{a b}$.

Proof. Let $S$ be the (ordered) set of points of $A$ of order 3, and let $k_{1}$ be the field of moduli of $(A, \mathscr{C}, \theta, S)$.

(i) $F^{\prime} \subset k_{1} \subset F_{a b}$

This follows from $[5,5.16]$

(ii) There is a model $\left(A_{1}, \mathscr{C}_{1}, \theta_{1}, S\right)$ for $(A, \mathscr{C}, \theta, S)$ defined over $k_{1}$.

It is easy to see that there is a finite normal extension $K$ of $k_{1}$ such that $(A, \mathscr{C}, \theta, S)$ is defined over $K$ and such that for every $\sigma \in \mathrm{Gal}\left(K / k_{1}\right)$ there is an isomorphism $\lambda_{\sigma}:(A, \mathscr{C}, \theta, S) \rightarrow\left(A^{\sigma}, \mathscr{C}^{\sigma}, \theta^{\sigma}, S^{\sigma}\right)$ defined over $K$. Let $\lambda_{\tau, \sigma}=\lambda_{\sigma-1}^{\sigma}$ for $\sigma, \tau \in \mathrm{Gal}\left(K / k_{1}\right)$. From the fact that Aut $(A, \mathscr{C}, \theta, S)=\{1\}[3, \S 21, \mathrm{Thm} 5]$ it follows that

$$
\begin{gathered}
\lambda_{\tau, \sigma}^{\rho}=\lambda_{\rho \tau, \rho \sigma} \\
\lambda_{\tau, \sigma} \lambda_{\sigma, \rho}=\lambda_{\tau, \rho}
\end{gathered}
$$

Received 8 June, 1972.

†This paper was written while the author was a visitor at King's College, London and the University of Nottingham, and was supported by the Science Research Council.

[BULl. LoNDON MATH. SOC., 4 (1972), 370-372] 
for all $\rho, \sigma, \tau \in \mathrm{Gal}\left(K / k_{1}\right)$. Assertion (ii) now follows from [7].

(iii) $A_{1}$, as in (ii) above, has all of its torsion points rational over $F_{a b}^{\prime}$ This is $[5,7.8 .8]$.

Regard now $(A, \mathscr{C}, \theta)$ as being defined over $k_{1}$ and satisfying (iii). If $k_{1}=k_{0}$ then the theorem is proved. If not, there is a field $k_{2}, k_{1} \supset k_{2} \supset k_{0} \supset F^{\prime}$, such that $k_{1} / k_{2}$ is Galois of prime degree $p$ (use (i)). Let $\sigma$ generate $\mathrm{Gal}\left(k_{1} / k_{2}\right)$ and let $\lambda:(A, C, \theta) \rightarrow\left(A^{\sigma}, C^{\sigma}, \theta^{\sigma}\right)$ be an isomorphism.

(iv) $\lambda$ is defined over $k_{1}$.

This is a consequence of [6, Thm 5, Pptn 1]. Alternatively it may be proved as follows. $a \mapsto a^{\sigma}$ is an isomorphism $V_{l} A \rightarrow V_{l} A^{\sigma}$ which commutes with the actions of $F$ and of $\mathrm{Gal}\left(\bar{k}_{1} / k_{1}\right)$ (use (iii)). But it is clear from [4, Cor 2 to Thm 5] that any homomorphism $V_{l} A \rightarrow V_{l} A$ which commutes with the action of $F$ commutes with the action of $\operatorname{Gal}\left(k_{1} / k_{1}\right)$. Thus $\lambda^{\tau}=\lambda$ for all $\tau \in \mathrm{Gal}\left(k_{1} / k_{1}\right)$ which proves (iv).

Write $v$ for the canonical isomorphism $\left(a^{\sigma p} \mapsto a\right):\left(A^{\sigma p}, \mathscr{C}^{\sigma p}, \theta^{\sigma p}\right) \rightarrow(A, \mathscr{C}, \theta)$. Then $\Lambda=v \lambda^{\sigma p-1} \ldots \lambda^{\sigma} \lambda$ is an automorphism of $(A, \mathscr{C}, \theta)$, and hence may be written as $\theta(\alpha)$ with $\alpha \in \mu(R)$ where $R=\theta^{-1}\left(\operatorname{End}_{\mathrm{c}}(A)\right)$ and $\mu(R)$ is the set of roots of unity in $R$.

(v) $\alpha$ is a pth power in $R$.

If $\mu$ is a homomorphism of abelian varieties we write $\mu_{l}$ for the corresponding map on the Tate groups $T_{l}$ (or $V_{l}$ ). The map $a \mapsto \lambda_{l}{ }^{-1}\left(a^{\sigma}\right): T_{l} A \rightarrow T_{l} A$ is $Z_{l}$-linear and commutes with the action of $\theta(R)$. By [4, Cor. 1 to Thm. 5] there exists an $\alpha_{l} \in R_{l}=R \otimes_{\mathbf{Z}} \mathbf{Z}_{l}$ such that $\lambda_{l}^{-1}\left(a^{\sigma}\right)=\theta\left(\alpha_{l}^{-1}\right)(a)$ all $a \in T_{l} A$. It follows that $\Lambda_{l}(a)=\theta\left(\alpha_{l}^{p}\right)(a)$ all $a \in T_{l} A$. Hence $\theta(\alpha)=\theta\left(\alpha_{l}^{p}\right)$, and so $\alpha$ is a $p$ th power in $R_{l}$ for all primes $l$. By class field theory, e.g. [1, $X]$, this implies that $\alpha$ is a $p$ th power in $F$, say $\alpha=\beta^{p}$. By using that $\alpha \in \mu(R)$ and is a $p$ th power in $R_{l}$ for all $l$, one gets that $\beta \in R_{l}$ for all $l$. But $R=\bigcap R_{l}$, and so $\beta \in R$.

Replace $\lambda$ by $\lambda \theta\left(\beta^{-1}\right)$, so that now $\Lambda=1$. Define $\lambda_{j, l}: A^{\sigma \prime} \rightarrow A^{\sigma J}$ by

$$
\lambda_{i, l}=\lambda^{\sigma j-1} \ldots \lambda^{\sigma l} \text {, }
$$

$0 \leqslant i \leqslant j \leqslant p-1$, and $\lambda_{j, i}=v^{\sigma j} \lambda_{j+p, i}, 0 \leqslant j \leqslant i \leqslant p-1$. Then $\lambda_{k, j} \lambda_{j, i}=\lambda_{k, i}$ and $\lambda_{j, i}^{\sigma}=\lambda_{j+1} i+1$ and so [7] there is an $\left(A_{2}, \mathscr{C}_{2}, \theta_{2}\right)$ defined over $k_{2}$ which is isomorphic to $(A, \mathscr{C}, \theta)$ over $k_{1}$. Note that $A_{2}$ will therefore also satisfy (iii). If $k_{2}=k$ the proof is complete. If not, the above process may be used to find an $\left(A_{3}, \mathscr{C}_{3}, \theta_{3}\right)$ over some $k_{3}, k_{2} \supset k_{3} \supset k, k_{2} \neq k_{3}$. By continuing in this way, one eventually obtains the desired result.

In order to state the two corollaries, consider $(A, \mathscr{C}, \theta)$ defined over some number field $k$, and let it be of type $(F, \Phi ; \mathfrak{a}, \zeta)$. Regard $F$ as a subfield of $\mathbf{C}$, write $I_{k}$ for the idèle group of $k$, put $I_{k, \infty}=k \otimes_{\mathbf{Q}} \mathbf{R} \subset I_{k}$, and write $I_{k}^{\infty}$ for the group of finite idèles of $k$, i.e. those whose component at any infinite prime is 1 . If $x \in I_{F}$, write $x_{1}$ for the component of $x$ corresponding to the infinite prime defined by the given embedding of $F \subset \mathbf{C}$. Then $\operatorname{det} \Phi^{\prime}$ defines a homomorphism $F^{\prime *} \rightarrow F^{*}$ and, since $k \supset F^{\prime}$, we 
get a homomorphism $g=\left(\operatorname{det} \Phi^{\prime}\right) N_{k / F^{\prime}}: k^{*} \rightarrow F^{*}$. This extends to a continuous homomorphism $I_{k} \rightarrow I_{F}$ which we also denote by $g$.

As explained in [6, p. 510], one obtains from $(A, \mathscr{C}, \theta)$ a Grössen-character $\psi: I_{k} \rightarrow \mathrm{C}^{*}$ such that,

(1) for all $x \in I_{k, \infty}, \psi(x)=g(x)_{1}{ }^{-1}$, and

(2) for all $x \in I_{k}^{\infty}, \psi(x) \in F^{*}, \psi(x) \overline{\psi(x)}=|x|_{0}$, and $\psi(x) \mathfrak{a}=g(x) \mathfrak{a}$, where $\overline{\psi(x)}$ is the complex conjugate of $\psi(x)$ and $|x|_{0}$ is the absolute norm of the ideal associated to $x$. Conversely, there is the following result.

COROLlary 1. Let $k$ be a finite extension of $F^{\prime}$. Any Grössen-character $\psi: I_{k} \rightarrow \mathbf{C}^{*}$ satisfying (1) and (2) arises from some $(A, \mathscr{C}, \theta)$ of type $(F, \Phi ; \mathfrak{a}, \zeta)$ defined over $k$.

Proof. Let $(A, \mathscr{C}, \theta)$ be any structure of type $(F, \Phi ; \mathfrak{a}, \zeta)$. It follows from $[5,5.16]$ that $k$ contains the field of moduli of $(A, \mathscr{C}, \theta)$ and so we may take $(A, \mathscr{C}, \theta)$ to be defined over $k$. Let $\psi^{\prime}$ be the Grössen-character arising from $(A, \mathscr{C}, \theta)$ and put $\chi=\psi / \psi^{\prime}$. By (1), $\chi$ is a Dirichlet character and so may be regarded as a character of $G=\mathrm{Gal}(K / k)$ for some finite abelian extension $K$ of $k$. Let $R_{\chi}$ be $R$ regarded as a $G$-module by defining $\sigma \alpha=\chi(\sigma) \alpha$ for $\sigma \in G, \alpha \in R$. Then, in the notation of [2, \$2], $\left(A^{\prime}, \mathscr{C}^{\prime}, \theta^{\prime}\right)$ with $A^{\prime}=R_{\chi} \otimes_{R} A$ and obvious $\theta^{\prime}$ and $\mathscr{C}^{\prime}$ is of type $(F, \Phi ; \mathfrak{a}, \zeta)$ and has Grössen-character $\chi \psi^{\prime}=\psi$.

Corollary 2. Let $k$ be a finite extension of $\mathbf{Q}$ and let $(F, \Phi ; \mathfrak{a}, \zeta)$ be a possible type for a structure $(A, \mathscr{C}, \theta)$. Then there is a Grössen-character $\psi: I_{k} \rightarrow \mathbf{C}^{*}$ satisfying (1) and (2) if and only if $k$ contains the field of moduli of some $(A, \mathscr{C}, \theta)$ of type $(F, \Phi ; \mathfrak{a}, \zeta)$.

Proof. The necessity follows from $[5,5.16]$ and the sufficiency from the theorem.

Remarks 1. In [6], Corollary 1 is proved directly and then, under certain hypotheses on $R$ ((5.2) loc. cit.), Shimura explicitly constructs a Grössen-character $\psi$ satisfying (1) and (2) and so deduces a weaker form of our Theorem 1.

2. Given $A$ and the map $\theta$ it is always possible to find a polarization $\mathscr{C}$ such that $\theta(F)^{\prime}=\theta(F)$ [5, p. 128]. Moreover [6, Pptn 4] the field of moduli of $(A, \mathscr{C}, \theta)$ is independent of the $\mathscr{C}$ chosen. Thus it makes sense to speak of the field of moduli of $(A, \theta)$, and then Theorem 1 implies that this is also the smallest field of definition of $(A, \theta)$.

\section{References}

1. E. Artin and J. Tate, Class field theory (Harvard University, 1961).

2. J. Milne, "On the arithmetic of abelian varieties ', Inventiones math. (to appear).

3. D. Mumford, Abelian varieties (Oxford University Press, London, 1970).

4. J.-P. Serre and J. Tate, " Good reduction of abelian varieties ", Ann. of Math., 88 (1968), 492-517.

5. G. Shimura, Introduction to the arithmetic theory of automorphic functions (Princeton U.P. 1971).

6. _- "On the zeta-function of an abelian variety with complex multiplication ", Ann. of Math., 94 (1971), 504-533.

7. A. Weil, “The field of definition of a variety", Amer. J. Math., 78 (1956), 509-524.

University of Michigan. 